\title{
Auxiliary material for: A general treatment of snow microstructure exemplified by an improved relation for the thermal conductivity
}

\author{
H. Löwe, F. Riche, M. Schneebeli \\ WSL Institute for Snow and Avalanche Research SLF, Davos, \\ Switzerland
}

\section{Classification of snow samples}

In the following we provide a detailed listing of used samples. The isothermal experiments are listed in Tab. 1. The time series of temperature gradient experiments (TGM-2, TGM17) were conducted in the $\mu$-CT and listed in Tab. 2,3. The temperature gradient experiments (DH-1, DH-2) were carried out in a metamorphism box and listed in Tab. 4. The set of 37 individual samples (DIV) comprising various types of snow is described in Tab. 5. Besides the ice volume fraction $\phi_{i}$ (density) (conservative error estimate: \pm 0.01 ) and the specific surface area (SSA) (conservative error estimate $\pm 10 \%$ ) we provide a measure of "grain size": The mean thickness Sp and Th of the ice matrix and pore space, respectively have been computed by the method [2] based on the inscription of spheres. The respective errors denote the standard deviation of the thickness distribution. In addition, a grain classification has been carried out from tomography images according to [1]. For time-lapse metamorphism experiments also the time step is given.

Table 1: Properties of the snow samples of isothermal experiments ISO-1 (upper part) and ISO-5 (lower part). ISC: international snow classification, Time in (weeks), Th: ice thickness (mm), Sp: pore thickness $(\mathrm{mm}), \phi_{i}$ : ice volume fraction, SSA: specific surface area $\left(\mathrm{mm}^{-1}\right)$.

\begin{tabular}{cccccc}
\hline ISC & Time & Th & Sp & $\phi_{i}$ & SSA \\
\hline DF dc & 0 & $0.07 \pm 0.02$ & $0.26 \pm 0.12$ & 0.167 & 43.51 \\
RG sr & 2 & $0.11 \pm 0.03$ & $0.33 \pm 0.14$ & 0.186 & 27.27 \\
RG sr & 5 & $0.14 \pm 0.03$ & $0.36 \pm 0.13$ & 0.198 & 22.71 \\
RG sr & 7 & $0.16 \pm 0.03$ & $0.37 \pm 0.14$ & 0.216 & 20.55 \\
RG sr & 10 & $0.18 \pm 0.03$ & $0.38 \pm 0.14$ & 0.221 & 19.51 \\
RG sr & 16 & $0.20 \pm 0.04$ & $0.40 \pm 0.15$ & 0.237 & 16.66 \\
RG lr & 31 & $0.25 \pm 0.05$ & $0.46 \pm 0.19$ & 0.249 & 14.04 \\
RG lr & 44 & $0.28 \pm 0.06$ & $0.46 \pm 0.18$ & 0.271 & 12.46 \\
RG lr & 47 & $0.29 \pm 0.06$ & $0.47 \pm 0.18$ & 0.277 & 14.19 \\
\hline DF dc & 0 & $0.08 \pm 0.03$ & $0.26 \pm 0.11$ & 0.176 & 37.79 \\
RG sr & 2 & $0.11 \pm 0.03$ & $0.31 \pm 0.12$ & 0.185 & 28.76 \\
RG sr & 5 & $0.12 \pm 0.04$ & $0.34 \pm 0.13$ & 0.187 & 25.45 \\
RG sr & 7 & $0.13 \pm 0.04$ & $0.36 \pm 0.14$ & 0.191 & 23.74 \\
RG sr & 10 & $0.14 \pm 0.04$ & $0.39 \pm 0.16$ & 0.190 & 22.19 \\
RG sr & 16 & $0.16 \pm 0.04$ & $0.44 \pm 0.20$ & 0.193 & 19.97 \\
RG sr & 31 & $0.19 \pm 0.04$ & $0.43 \pm 0.16$ & 0.218 & 17.16 \\
RG sr & 44 & $0.21 \pm 0.05$ & $0.46 \pm 0.19$ & 0.224 & 16.79 \\
RG sr & 47 & $0.21 \pm 0.05$ & $0.46 \pm 0.19$ & 0.228 & 16.36 \\
\hline
\end{tabular}


Table 2: Properties of the snow samples of TGM-17 temperature gradient experiment. ISC: international snow classification, Time in (days), Th: ice thickness ( $\mathrm{mm})$, Sp: pore thickness $(\mathrm{mm}), \phi_{i}$ : ice volume fraction, SSA: specific surface area $\left(\mathrm{mm}^{-1}\right)$.

\begin{tabular}{|c|c|c|c|c|c|}
\hline ISC & Time & $\mathrm{Th}$ & $\mathrm{Sp}$ & $\overline{\phi_{i}}$ & SSA \\
\hline RG sr & $\overline{0.0}$ & $0.13 \pm 0.03$ & $0.24 \pm 0.09$ & 0.289 & 21.70 \\
\hline RG sr & 0.3 & $0.14 \pm 0.03$ & $0.25 \pm 0.09$ & 0.288 & 21.46 \\
\hline RG sr & 0.7 & $0.14 \pm 0.04$ & $0.25 \pm 0.10$ & 0.287 & 21.05 \\
\hline RG sr & 1.0 & $0.14 \pm 0.04$ & $0.25 \pm 0.10$ & 0.287 & 20.60 \\
\hline RG sr & 1.3 & $0.15 \pm 0.04$ & $0.26 \pm 0.10$ & 0.289 & 20.13 \\
\hline RG sr & 1.7 & $0.15 \pm 0.04$ & $0.26 \pm 0.10$ & 0.288 & 19.79 \\
\hline RG sr & 2.0 & $0.16 \pm 0.04$ & $0.27 \pm 0.10$ & 0.292 & 19.18 \\
\hline$R G \times f$ & 2.3 & $0.16 \pm 0.05$ & $0.27 \pm 0.11$ & 0.290 & 18.87 \\
\hline RG xf & 2.7 & $0.16 \pm 0.05$ & $0.28 \pm 0.11$ & 0.291 & 18.47 \\
\hline $\mathrm{FC} \mathrm{xr}$ & 3.0 & $0.16 \pm 0.05$ & $0.28 \pm 0.11$ & 0.292 & 18.13 \\
\hline $\mathrm{FC} \mathrm{xr}$ & 3.3 & $0.17 \pm 0.05$ & $0.28 \pm 0.11$ & 0.292 & 17.83 \\
\hline $\mathrm{FC} \mathrm{xr}$ & 3.7 & $0.17 \pm 0.05$ & $0.29 \pm 0.11$ & 0.292 & 17.49 \\
\hline $\mathrm{FC} \mathrm{xr}$ & 4.0 & $0.17 \pm 0.06$ & $0.30 \pm 0.11$ & 0.292 & 17.14 \\
\hline FC so & 4.3 & $0.18 \pm 0.06$ & $0.30 \pm 0.12$ & 0.292 & 16.77 \\
\hline FC so & 4.7 & $0.18 \pm 0.06$ & $0.31 \pm 0.12$ & 0.292 & 16.55 \\
\hline FC so & 5.0 & $0.18 \pm 0.06$ & $0.31 \pm 0.12$ & 0.293 & 16.20 \\
\hline FC so & 5.3 & $0.18 \pm 0.06$ & $0.32 \pm 0.13$ & 0.294 & 15.98 \\
\hline FC so & 5.7 & $0.18 \pm 0.06$ & $0.32 \pm 0.13$ & 0.294 & 15.77 \\
\hline FC so & 6.0 & $0.19 \pm 0.07$ & $0.33 \pm 0.13$ & 0.293 & 15.55 \\
\hline FC so & 6.3 & $0.19 \pm 0.07$ & $0.33 \pm 0.13$ & 0.294 & 15.39 \\
\hline FC so & 6.7 & $0.19 \pm 0.07$ & $0.34 \pm 0.14$ & 0.294 & 15.18 \\
\hline FC so & 7.0 & $0.19 \pm 0.07$ & $0.34 \pm 0.14$ & 0.294 & 15.08 \\
\hline FC so & 7.3 & $0.19 \pm 0.07$ & $0.35 \pm 0.14$ & 0.295 & 14.96 \\
\hline FC so & 7.7 & $0.19 \pm 0.07$ & $0.35 \pm 0.14$ & 0.293 & 14.99 \\
\hline FC so & 8.0 & $0.19 \pm 0.07$ & $0.36 \pm 0.15$ & 0.293 & 14.90 \\
\hline FC so & 8.3 & $0.19 \pm 0.07$ & $0.36 \pm 0.15$ & 0.293 & 14.85 \\
\hline FC so & 8.7 & $0.19 \pm 0.07$ & $0.36 \pm 0.15$ & 0.293 & 14.81 \\
\hline FC so & 9.0 & $0.19 \pm 0.07$ & $0.36 \pm 0.15$ & 0.293 & 14.76 \\
\hline FC so & 9.3 & $0.19 \pm 0.07$ & $0.37 \pm 0.16$ & 0.294 & 14.67 \\
\hline $\mathrm{DH} \mathrm{cp}$ & 9.7 & $0.19 \pm 0.07$ & $0.37 \pm 0.16$ & 0.295 & 14.58 \\
\hline DH cp & 10.0 & $0.18 \pm 0.07$ & $0.36 \pm 0.16$ & 0.295 & 14.86 \\
\hline $\mathrm{DH} \mathrm{cp}$ & 10.3 & $0.18 \pm 0.07$ & $0.37 \pm 0.16$ & 0.294 & 14.60 \\
\hline $\mathrm{DH} \mathrm{cp}$ & 10.7 & $0.19 \pm 0.07$ & $0.38 \pm 0.16$ & 0.294 & 14.51 \\
\hline DH cp & 11.0 & $0.19 \pm 0.07$ & $0.38 \pm 0.16$ & 0.295 & 14.38 \\
\hline $\mathrm{DH} \mathrm{cp}$ & 11.3 & $0.18 \pm 0.07$ & $0.38 \pm 0.16$ & 0.293 & 14.53 \\
\hline $\mathrm{DH} \mathrm{cp}$ & 11.7 & $0.19 \pm 0.07$ & $0.38 \pm 0.17$ & 0.295 & 14.34 \\
\hline DH cp & 12.0 & $0.19 \pm 0.07$ & $0.38 \pm 0.17$ & 0.296 & 14.31 \\
\hline $\mathrm{DH} \mathrm{cp}$ & 12.3 & $0.19 \pm 0.07$ & $0.38 \pm 0.17$ & 0.296 & 14.26 \\
\hline $\mathrm{DH} \mathrm{cp}$ & 12.7 & $0.19 \pm 0.07$ & $0.39 \pm 0.17$ & 0.295 & 14.19 \\
\hline $\mathrm{DH} \mathrm{cp}$ & 13.0 & $0.19 \pm 0.07$ & $0.39 \pm 0.17$ & 0.295 & 14.09 \\
\hline $\mathrm{DH} \mathrm{cp}$ & 13.3 & $0.19 \pm 0.07$ & $0.40 \pm 0.17$ & 0.296 & 13.99 \\
\hline $\mathrm{DH} \mathrm{cp}$ & 13.7 & $0.19 \pm 0.07$ & $0.40 \pm 0.17$ & 0.295 & 13.96 \\
\hline DH cp & 14.0 & $0.19 \pm 0.07$ & $0.41 \pm 0.18$ & 0.296 & 13.78 \\
\hline DH cp & 14.3 & $0.19 \pm 0.07$ & $0.41 \pm 0.18$ & 0.295 & 13.78 \\
\hline $\mathrm{DH} \mathrm{cp}$ & 14.7 & $0.19 \pm 0.07$ & $0.41 \pm 0.18$ & 0.294 & 13.77 \\
\hline DH cp & 15.0 & $0.19 \pm 0.07$ & $0.41 \pm 0.18$ & 0.294 & 13.71 \\
\hline
\end{tabular}




\begin{tabular}{llllll} 
DH cp & 15.3 & $0.20 \pm 0.08$ & $0.42 \pm 0.18$ & 0.294 & 13.62 \\
DH cp & 15.7 & $0.20 \pm 0.08$ & $0.42 \pm 0.19$ & 0.294 & 13.57 \\
DH cp & 16.0 & $0.20 \pm 0.08$ & $0.42 \pm 0.19$ & 0.296 & 13.40 \\
\hline
\end{tabular}

Table 3: Properties of the snow samples of TGM-2 temperature gradient experiment. ISC: international snow classification, Time in (days), Th: ice thickness $(\mathrm{mm})$, Sp: pore thickness $(\mathrm{mm}), \phi_{i}$ : ice volume fraction, SSA: specific surface area $\left(\mathrm{mm}^{-1}\right)$.

\begin{tabular}{cccccc}
\hline ISC & Time & Th & Sp & $\phi_{i}$ & SSA \\
\hline DF dc & 0.0 & $0.10 \pm 0.04$ & $0.26 \pm 0.11$ & 0.218 & 28.95 \\
DF dc & 0.1 & $0.10 \pm 0.04$ & $0.27 \pm 0.12$ & 0.219 & 28.66 \\
DF dc & 0.4 & $0.11 \pm 0.04$ & $0.27 \pm 0.12$ & 0.217 & 28.05 \\
DF dc & 0.6 & $0.11 \pm 0.04$ & $0.29 \pm 0.13$ & 0.218 & 27.25 \\
RG sr & 0.9 & $0.11 \pm 0.04$ & $0.29 \pm 0.13$ & 0.219 & 26.51 \\
RG sr & 1.1 & $0.12 \pm 0.05$ & $0.30 \pm 0.13$ & 0.220 & 25.70 \\
RG sr & 1.4 & $0.12 \pm 0.05$ & $0.31 \pm 0.14$ & 0.221 & 24.87 \\
RG sr & 1.4 & $0.12 \pm 0.05$ & $0.32 \pm 0.14$ & 0.223 & 24.16 \\
RG xf & 1.9 & $0.13 \pm 0.05$ & $0.32 \pm 0.14$ & 0.226 & 23.57 \\
RG xf & 2.1 & $0.13 \pm 0.05$ & $0.34 \pm 0.15$ & 0.225 & 23.00 \\
RG xf & 2.4 & $0.13 \pm 0.06$ & $0.34 \pm 0.15$ & 0.228 & 22.42 \\
RG xf & 2.6 & $0.14 \pm 0.06$ & $0.35 \pm 0.16$ & 0.231 & 21.93 \\
FC so & 2.9 & $0.14 \pm 0.06$ & $0.36 \pm 0.16$ & 0.230 & 21.62 \\
FC so & 3.1 & $0.14 \pm 0.06$ & $0.37 \pm 0.17$ & 0.229 & 21.35 \\
FC so & 3.4 & $0.14 \pm 0.06$ & $0.37 \pm 0.17$ & 0.231 & 20.92 \\
FC so & 3.6 & $0.15 \pm 0.06$ & $0.38 \pm 0.17$ & 0.232 & 20.34 \\
FC so & 3.9 & $0.15 \pm 0.07$ & $0.39 \pm 0.17$ & 0.233 & 19.89 \\
FC so & 4.7 & $0.15 \pm 0.07$ & $0.41 \pm 0.19$ & 0.231 & 19.06 \\
DH cp & 4.9 & $0.15 \pm 0.07$ & $0.41 \pm 0.19$ & 0.234 & 18.90 \\
DH cp & 5.1 & $0.16 \pm 0.07$ & $0.42 \pm 0.19$ & 0.235 & 18.60 \\
DH cp & 5.4 & $0.16 \pm 0.07$ & $0.43 \pm 0.20$ & 0.236 & 18.32 \\
DH cp & 5.6 & $0.16 \pm 0.07$ & $0.43 \pm 0.20$ & 0.237 & 18.05 \\
DH cp & 5.9 & $0.16 \pm 0.07$ & $0.43 \pm 0.20$ & 0.238 & 17.83 \\
DH cp & 6.1 & $0.17 \pm 0.07$ & $0.44 \pm 0.20$ & 0.241 & 17.47 \\
DH cp & 6.4 & $0.17 \pm 0.07$ & $0.45 \pm 0.21$ & 0.241 & 17.12 \\
DH cp & 6.6 & $0.17 \pm 0.08$ & $0.44 \pm 0.20$ & 0.244 & 16.94 \\
DH cp & 6.9 & $0.17 \pm 0.08$ & $0.44 \pm 0.20$ & 0.246 & 16.81 \\
DH cp & 7.1 & $0.17 \pm 0.07$ & $0.44 \pm 0.20$ & 0.248 & 16.61 \\
DH cp & 7.4 & $0.17 \pm 0.08$ & $0.45 \pm 0.20$ & 0.251 & 16.43 \\
DH cp & 7.6 & $0.18 \pm 0.08$ & $0.47 \pm 0.22$ & 0.244 & 16.24 \\
DH cp & 7.9 & $0.18 \pm 0.08$ & $0.47 \pm 0.21$ & 0.245 & 16.09 \\
DH cp & 8.1 & $0.18 \pm 0.08$ & $0.47 \pm 0.22$ & 0.246 & 15.97 \\
DH cp & 8.4 & $0.18 \pm 0.09$ & $0.48 \pm 0.22$ & 0.246 & 15.75 \\
DH cp & 8.6 & $0.18 \pm 0.09$ & $0.48 \pm 0.22$ & 0.248 & 15.70 \\
DH cp & 8.9 & $0.18 \pm 0.09$ & $0.48 \pm 0.23$ & 0.252 & 15.47 \\
DH cp & 9.1 & $0.19 \pm 0.09$ & $0.50 \pm 0.24$ & 0.254 & 15.24 \\
DH cp & 9.4 & $0.19 \pm 0.09$ & $0.50 \pm 0.24$ & 0.258 & 15.16 \\
DH cp & 9.6 & $0.19 \pm 0.09$ & $0.50 \pm 0.24$ & 0.258 & 15.05 \\
DH cp & 9.9 & $0.19 \pm 0.09$ & $0.51 \pm 0.25$ & 0.259 & 15.01 \\
DH cp & 10.1 & $0.19 \pm 0.09$ & $0.51 \pm 0.25$ & 0.255 & 14.75
\end{tabular}




\begin{tabular}{llllll} 
DH cp & 10.4 & $0.20 \pm 0.09$ & $0.53 \pm 0.26$ & 0.256 & 14.32 \\
DH cp & 10.6 & $0.20 \pm 0.09$ & $0.53 \pm 0.26$ & 0.256 & 14.23 \\
DH cp & 10.9 & $0.20 \pm 0.10$ & $0.55 \pm 0.27$ & 0.255 & 14.12 \\
DH cp & 11.1 & $0.20 \pm 0.10$ & $0.55 \pm 0.28$ & 0.256 & 14.07 \\
DH cp & 11.4 & $0.20 \pm 0.10$ & $0.56 \pm 0.28$ & 0.257 & 13.97 \\
DH cp & 11.6 & $0.20 \pm 0.10$ & $0.55 \pm 0.27$ & 0.258 & 13.97 \\
DH cp & 11.9 & $0.21 \pm 0.10$ & $0.56 \pm 0.28$ & 0.257 & 13.94 \\
\hline
\end{tabular}

Table 4: Properties of the snow samples DH-1 (Upper part) and DH-2 (lower part). ISC: international snow classification, Time in (weeks), Th: ice thickness (mm), Sp: pore thickness (mm), $\phi_{i}$ : ice volume fraction, SSA: specific surface area $\left(\mathrm{mm}^{-1}\right)$.

\begin{tabular}{cccccr}
\hline ISC & Time & Th & Sp & $\phi_{i}$ & SSA \\
\hline DF dc & 0 & $0.14 \pm 0.04$ & $0.37 \pm 0.13$ & 0.196 & 22.13 \\
RG xf & 1 & $0.15 \pm 0.05$ & $0.34 \pm 0.12$ & 0.224 & 20.61 \\
RG xf & 2 & $0.16 \pm 0.06$ & $0.44 \pm 0.17$ & 0.190 & 19.57 \\
DH cp & 4 & $0.19 \pm 0.09$ & $0.48 \pm 0.20$ & 0.225 & 16.26 \\
DH cp & 6 & $0.25 \pm 0.11$ & $0.58 \pm 0.24$ & 0.277 & 11.50 \\
DH cp & 12.5 & $0.29 \pm 0.14$ & $0.69 \pm 0.33$ & 0.324 & 9.29 \\
\hline DF bk & 0 & $0.15 \pm 0.05$ & $0.31 \pm 0.15$ & 0.281 & 19.96 \\
RG wp & 1 & $0.16 \pm 0.05$ & $0.27 \pm 0.11$ & 0.332 & 18.17 \\
RG xf & 4 & $0.20 \pm 0.08$ & $0.34 \pm 0.15$ & 0.360 & 14.08 \\
FC so & 6 & $0.2 \pm 0.10$ & $0.46 \pm 0.22$ & 0.332 & 12.23 \\
DH cp & 11.5 & $0.28 \pm 0.15$ & $0.51 \pm 0.27$ & 0.406 & 9.35 \\
\hline
\end{tabular}

Table 5: Properties of diverse snow samples (DIV). ISC: international snow classification, Th: ice thickness $(\mathrm{mm})$, Sp: pore thickness $(\mathrm{mm}), \phi_{i}$ : ice volume fraction, SSA: specific surface area $\left(\mathrm{mm}^{-1}\right)$.

\begin{tabular}{cccccr}
\hline ISC & Time & Th & Sp & $\phi_{i}$ & SSA \\
\hline DH cp & - & $0.17 \pm 0.07$ & $0.49 \pm 0.23$ & 0.260 & 15.99 \\
DH cp & - & $0.16 \pm 0.07$ & $0.47 \pm 0.22$ & 0.260 & 16.37 \\
DH cp & - & $0.20 \pm 0.10$ & $0.62 \pm 0.33$ & 0.290 & 12.97 \\
DH cp & - & $0.17 \pm 0.07$ & $0.54 \pm 0.32$ & 0.310 & 14.05 \\
DH cp & - & $0.19 \pm 0.10$ & $0.51 \pm 0.28$ & 0.330 & 13.51 \\
DH cp & - & $0.23 \pm 0.14$ & $0.93 \pm 0.53$ & 0.230 & 12.28 \\
DH cp & - & $0.25 \pm 0.13$ & $0.78 \pm 0.37$ & 0.250 & 11.31 \\
DH cp & - & $0.29 \pm 0.20$ & $0.82 \pm 0.44$ & 0.280 & 10.51 \\
DH cp & - & $0.20 \pm 1.76$ & $0.07 \pm 1.27$ & 0.122 & 12.90 \\
FC so & - & $0.41 \pm 0.18$ & $0.71 \pm 0.29$ & 0.340 & 7.11 \\
FC so & - & $0.26 \pm 0.09$ & $0.54 \pm 0.21$ & 0.270 & 11.65 \\
FC so & - & $0.25 \pm 0.42$ & $0.07 \pm 0.15$ & 0.302 & 12.71 \\
FC xr & - & $0.18 \pm 0.40$ & $0.05 \pm 0.14$ & 0.242 & 17.05 \\
MF cl & - & $0.31 \pm 0.12$ & $0.63 \pm 0.29$ & 0.300 & 9.40 \\
MF cl & - & $0.31 \pm 0.11$ & $0.40 \pm 0.17$ & 0.410 & 9.62 \\
MF cl & - & $0.35 \pm 0.13$ & $0.80 \pm 0.35$ & 0.290 & 8.21 \\
MF cl & - & $0.21 \pm 0.06$ & $0.34 \pm 0.13$ & 0.320 & 14.72 \\
MF cl & - & $0.21 \pm 0.06$ & $0.31 \pm 0.12$ & 0.330 & 15.14
\end{tabular}




\begin{tabular}{lllllr} 
RG lr & - & $0.26 \pm 0.09$ & $0.49 \pm 0.20$ & 0.290 & 11.89 \\
RG sr & - & $0.12 \pm 0.03$ & $0.31 \pm 0.11$ & 0.170 & 29.03 \\
RG sr & - & $0.13 \pm 0.03$ & $0.28 \pm 0.10$ & 0.210 & 26.39 \\
RG sr & - & $0.12 \pm 0.03$ & $0.33 \pm 0.12$ & 0.170 & 27.15 \\
RG sr & - & $0.13 \pm 0.03$ & $0.30 \pm 0.11$ & 0.200 & 25.50 \\
RG sr & - & $0.13 \pm 0.04$ & $0.56 \pm 0.34$ & 0.130 & 25.09 \\
RG sr & - & $0.12 \pm 0.03$ & $0.34 \pm 0.13$ & 0.180 & 26.78 \\
RG sr & - & $0.14 \pm 0.04$ & $0.36 \pm 0.14$ & 0.190 & 23.41 \\
RG sr & - & $0.13 \pm 0.03$ & $0.32 \pm 0.12$ & 0.210 & 24.90 \\
RG sr & - & $0.10 \pm 0.03$ & $0.24 \pm 0.09$ & 0.210 & 31.34 \\
RG sr & - & $0.13 \pm 0.04$ & $0.29 \pm 0.11$ & 0.220 & 24.85 \\
RG sr & - & $0.19 \pm 0.41$ & $0.07 \pm 0.16$ & 0.256 & 16.16 \\
RG sr & - & $0.22 \pm 0.30$ & $0.05 \pm 0.13$ & 0.363 & 14.47 \\
RG sr & - & $0.16 \pm 0.27$ & $0.03 \pm 0.09$ & 0.294 & 19.89 \\
RG sr & - & $0.34 \pm 0.81$ & $0.13 \pm 0.36$ & 0.261 & 8.84 \\
RG xf & - & $0.18 \pm 0.38$ & $0.04 \pm 0.14$ & 0.246 & 17.63 \\
PP pp & - & $0.04 \pm 0.03$ & $0.30 \pm 0.14$ & 0.113 & 62.35 \\
PP pp & - & $0.03 \pm 0.01$ & $0.27 \pm 0.15$ & 0.067 & 103.19 \\
PP pp & - & $0.03 \pm 0.01$ & $0.26 \pm 0.13$ & 0.078 & 90.82 \\
RG sr & - & $0.13 \pm 0.04$ & $0.30 \pm 0.11$ & 0.194 & 25.91 \\
DF dc & - & $0.05 \pm 0.02$ & $0.16 \pm 0.04$ & 0.085 & 65.12 \\
DF dc & - & $0.06 \pm 0.02$ & $0.16 \pm 0.04$ & 0.144 & 56.54 \\
FC so & - & $0.19 \pm 0.08$ & $0.35 \pm 0.17$ & 0.316 & 15.69 \\
FC so & - & $0.21 \pm 0.07$ & $0.24 \pm 0.11$ & 0.459 & 13.14 \\
\hline
\end{tabular}

\section{References}

[1] Fierz, C., R. L. Armstrong, Y. Durand, P. Etchevers, E. Greene, D. M. McClung, K. Nishimura, P. K. Satyawali, and S. Sokratov, The international classification for seasonal snow on the ground, IHP-VII Technical Documents in Hydrology, 83, IACS Contribution (1), UNESCO-IHP, Paris, 2009.

[2] Hildebrand, T., and P. Ruegsegger, A new method for the model-independent assessment of thickness in three-dimensional images, J. Microscopy, 185(1), 67-75, 1997. 